
PRIMARY SJÖGREN'S SYNDROME PATIENTS

K. Ben Abdelghani, M. Chammakhi, A. Fazaa, K. Ouenniche, S. Kassab, S. Chekili, A. Lataar. rheumatology, Mongi Slim Hospital, La Marsa, Tunisia

Background: Primary Sjögren's Syndrome is an auto-immune disease characterised by dryness of the eyes and the oral cavity. Musculoskeletal manifestations are common. However, the underlying mechanism remains often unknown.

Objectives: The aim of the current study was to describe subclinical entheseal involvement in patients with Primary Sjögren's Syndrome via ultrasound, to calculate a modified Madrid sonography enthesitis index and to compare it with a group of healthy controls.

Methods: The study was conducted in the rheumatology department of Mongi Slim hospital in Tunisia, between June 2015 and December 2017, including 25 patients with Primary Sjögren's Syndrome and 25 healthy sex- and age-matched controls. Cases were defined according to the American-European Consensus Criteria for Sjögren's Syndrome. All the included subjects underwent an enthesis ultrasound exploration (EsaoteMyLab 60 machine and a 13-18 MHz linear array transducer) by a rheumatogist experimented in ultrasound. Five enthesis locations bilaterally (distal Achilles tendon, distal and proximal patellar ligaments, distal quadriceps, and brachial triceps tendons) in each patient were explored. The following elemental lesions of enthesis were evaluated: thickning, presence of calcifications, erosions, enthesophyte, loss of fibrillar pattern and power Doppler signal. The calculated index was compared by Mann-Whitney $U$ test between cases and controls. The significance level was set at $5 \%$.

Results: In our study population, the median age was $53.2 \pm 11.3$ years and the median body mass index was $29 . \pm 6.4 \mathrm{~kg} / \mathrm{m}^{2}$;. All included subjects were female. The ultrasound abnormalities in the Primary Sjögren's Syndrome were as follows: erosions in $19.2 \%$ of cases, enthesophytes in $16.4 \%$ of cases, calcifications in $6 \%$ of cases, hypoechogeneity in $2.8 \%$ of cases, thickning in $2.4 \%$ of cases, power Doppler signal in $1.6 \%$ of cases and loss of fibrillar pattern in $1.2 \%$ of cases. The total enthesitis index was 4,96 $\pm 2,59$ among cases and 5,72 $\pm 2,92$ among healthy control subjects with no statistically significant difference. Considering each affected enthesis, cases had no significantly higher scores than controls.

Conclusions: Our study did not find a significant enthesal involvement among patients with Primary Sjögren's Syndrome that could explain the chronic indefinable pain. The diagnosis of an associated fibromyalgia should be kept in mind.

Disclosure of Interest: None declared

DOI: 10.1136/annrheumdis-2018-eular.7424

\section{AB0602 ANTIPHOSPHOLIPID SYNDROME COMPONENTS IN PATIENTS WITH CORONARY HEART DISEASE}

M. Nazarova ${ }^{1}$, M. Stanislavchuk ${ }^{2}$, L. Burdeina ${ }^{3} .{ }^{1} P h D$-student of the internal medicine chair $\# 1 ;{ }^{2}$ Professor, the head of the internal medicine chair $\# 1 ;{ }^{3} \mathrm{PhD}$ of the internal medicine chair \#1, National Pirogov Memorial Medical University, Vinnytsa, Ukraine

Background: Antiphospholipid antibodies (aPL), such as anticardiolipin antibodies $(\mathrm{aCL})$, are the immunological markers of the antiphospholipid syndrome (APS). ${ }^{1}$ The aPL are found in association with venous and/or arterial thrombosis. Myocardial infarction (MI) is usually related to atherosclerosis and thrombosis of coronary arteries. $^{2}$ The clinical significance of aPL in MI, however, has not yet been well established.

Objectives: To evaluate the presence and levels of aPL in patients with history Ml.

Methods: 50 patients $(100 \%$ male) with average age $49.5 \pm 6.09(M \pm S D)$ years with history of MI were examined. Serum IgG aPL (anti-cardiolipin, -phosphatidylserine, phosphatidylinositol, -phosphatidylacetate) were determined by enzymelinked immunosorbent assay (ELSSA).

Results: IgG isotype aPL were detected in $26(52 \%)$ patients with the history of $\mathrm{Ml}$ and 24 patients were negative.

The average age $(M \pm S D)$ of aPL positive patients was $44.1 \pm 5.00$ years and of aPL negative patients was $47.7 \pm 4.83$ years $(p<0.01)$. The difference comprises more than 3,5 years.

Patients with recurrent $\mathrm{MI}$ (two and more) had higher level of IgG aPL, then patients with one Ml $(18,0 \pm 7,53$ v.s. $11,5 \pm 4,21 \mathrm{GPL}-\mathrm{U} / \mathrm{ml})$. The difference is significant $(p<0.05)$

Conclusions: Determined younger age of the first MI in aPL positive patients and higher level of $\mathrm{IgG}$ aPL in patients with recurrent Ml indicate the possible involvement of the autoimmune factor in the pathogenesis of Ml. This proves the necessity for further research in this direction.

\section{REFERENCES:}

[1] Miyakis S, Lockshin MD, Atsumi T, et al. International consensus statement on an update of the classification criteria for definite antiphospholipid syndrome (APS). J Thromb Haemost. 2006:4:295-306.
[2] Anand SS, Islam S, Rosengren A, Franzosi MG, Steyn K, Yusufali AH, et al. Patterns of alcohol consumption and myocardial infarction risk: observations from 52 countries in the INTERHEART case-control study. Eur Heart J 2008;29;932-940.

Disclosure of Interest: None declared

DOI: 10.1136/annrheumdis-2018-eular.5801

\section{AB0603 1 FRAGMENTED QRS IN PATIENTS WITH SYSTEMIC LUPUS ERYTHEMATOSUS: RELATION TO THE DISEASE ACTIVITY: A CROSS-SECTIONAL STUDY}

M. Hosonuma, N. Yajima. Division of Rheumatology, Department of Medicine, Showa University School of Medicine, Tokyo, Japan

Background: Cardiovascular disease is an important contributor to mortality in Systemic Lupus Erythematosus (SLE) ${ }^{1}$. Fragmented QRS (fQRS) is an easily evaluated non-invasive electrocardiographic parameter defined as additional spikes within the QRS complex ${ }^{2}$. fQRS can represent conduction disturbance and a predictor of cardiac events ${ }^{3}$. Even in a patient with SLE, it has reported that the prevalence of fQRS appears to be higher than in controls ${ }^{4}$. However, no clinical studies have investigated the prevalence at the time of diagnosis. In addition, there is no report that examined the association of disease activity of SLE and fQRS.

Objectives: This study aimed to assess the relationship between disease activity of SLE and fQRS in Japanese SLE patients at the time of diagnosis. We hypothesised that the frequency of fQRS on ECGs would be greater in SLE patients with high disease activity.

Methods: The study design was a cross-sectional study. The participants were SLE patients who diagnosed at Showa University Hospital and Showa University Koto Toyosu Hospital from January 2010 to December 2017. The participants who satisfied American College of Rheumatology (ACR) criteria were included. The patients with already treatment at the time of an ECG measurement, cardiovascular disease, history of arrhythmia, cardiomyopathy, rheumatoid arthritis, systemic sclerosis were excluded. The exposure was the appearance of fQRS. The primary outcome was Systemic Lupus Erythematosus Disease Activity Index 2000 (SLEDAI-2K). The secondary outcomes were the complement level, the ds DNA antibody level and the organ involvement. In the main analysis, a multiple regression analysis was conducted to assess the association between fQRS and SLE activity adjusted for age, sex and period from the estimated date of onset to the date of diagnosis. In the secondary analysis, a multiple regression analysis was conducted to analysis the association between fQRS and the serological activity of SLE (the complement level, the ds-DNA antibody level) and the organ involvement under the same conditions as above.

Results: In total, 45 participants were enrolled. The mean age was 42.3 years, and 37 (82\%) were female. The median SLEDAI-2K was 14 [IQR, 10 to 20]. The median period from the estimated date of onset to the date of diagnosis was 3 months [IQR, 2 to 14.5]. 25 patients (56\%) had fQRS. In the main analysis, the regression coefficients [95\% Cl] of fQRS for SLEDAI were 2.99 [ 1.15 to 4.84 $\mathrm{p}=0.002$ ] with reference to non-fQRS. In the secondary analysis, there were no significant associations between fQRS and the blood test or the organ involvement.

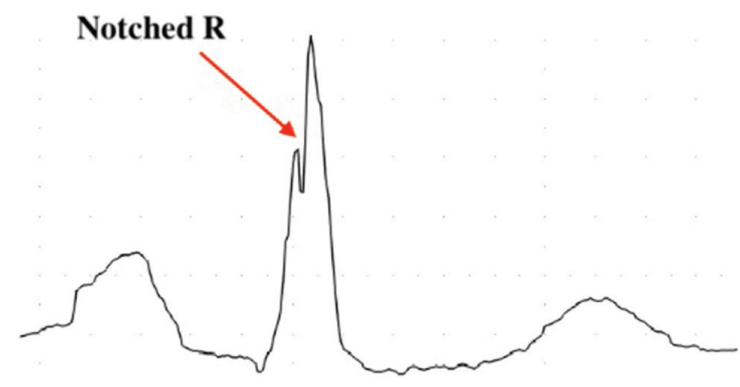

Fragmented QRS on 12-lead 